\title{
Are patient ratings of chronic pain services related to treatment outcome?
}

\author{
Gabriel Tan, PhD, ABPP; ${\text { 1-2* Mark P. Jensen, PhD; }{ }^{3} \text { John I. Thornby, PhD; }{ }^{1-2} \text { Karen O. Anderson, PhD }}^{4}$ \\ ${ }^{1}$ Michael E. DeBakey Department of Veterans Affairs Medical Center, Houston, TX; ${ }^{2}$ Baylor College of Medicine, \\ Houston, TX; ${ }^{3}$ Department of Rehabilitation Medicine, University of Washington, Seattle, WA; ${ }^{4}$ Department of \\ Symptom Research, The University of Texas M. D. Anderson Cancer Center, Houston, TX
}

\begin{abstract}
Consumer ratings of satisfaction with treatment are rarely used as measures of treatment outcome. This study examined the relationships between service ratings and psychometric outcomes of patients receiving pain-management services in a tertiary teaching hospital. A group of 122 patients who completed a multidisciplinary pain-management program rated their satisfaction with and effectiveness of services received and changes in their pain condition and quality of life (QOL). They also completed pre- and posttreatment measures of pain severity, pain interference, depression, and disability. Pain severity, pain interference, and depression significantly decreased following treatment. The patients' ratings of services were significantly associated with outcome measures. Pre- to posttreatment changes in pain severity and pain interference were associated with treatment satisfaction and effectiveness, improvement in pain condition, and QOL. Pre- to posttreatment change in disability was significantly related to ratings of treatment effectiveness, improvement in pain condition, and quality of life. The findings suggest that pain intensity, pain interference, and disability are important outcome dimensions of pain-management programs.
\end{abstract}

Key words: chronic pain, chronic pain treatment, depression, disability, multidisciplinary pain treatment, outcome assessment, pain interference, patient satisfaction, ratings of pain services, rehabilitation, treatment outcome, treatment satisfaction.

\section{INTRODUCTION}

In this age of accountability, patient feedback, usually on satisfaction with services received, has become a staple for many hospitals and health service providers. An advantage of patient satisfaction ratings is that they offer a low-cost, convenient assessment of the patient's perception of benefit from services. Numerous studies have found that a good patient-provider relationship may lead to a number of positive outcomes [1].

Despite the popularity and convenience of patient satisfaction ratings, very little research has studied how they relate to changes in specific treatment outcome domains; what little research has been performed suggests that the association between patient satisfaction and functional treatment outcomes varies. For example, one study found that treatment satisfaction was poorly related to objective functional scores among patients who received limited wrist fusion [2]. Although most patients

\footnotetext{
Abbreviations: ANOVA $=$ analysis of variance, CES-D = Center for Epidemiologic Studies Depression Scale, GED = general equivalency diploma, IPMP = Integrated Pain Management Program, MANOVA = multivariate ANOVA, PM\&R = physical medicine and rehabilitation, QOL = quality of life, RMDQ = Roland-Morris Disability Questionnaire, VAMC = Department of Veterans Affairs medical center, WHYMPI = West Haven-Yale Multidimensional Pain Inventory.

*Address all correspondence to Gabriel Tan, PhD, ABPP; Pain Section, Anesthesiology (145), Michael E. DeBakey VA Medical Center, 2002 Holcombe Boulevard, Houston, TX 77030; 713-794-8794; fax: 713-794-7674.

Email: tan.gabriel@med.va.gov

DOI: $10.1682 / J R R D .2004 .10 .0128$
} 
in this study indicated that they were satisfied with the results of their surgery, their objective posttreatment wrist-functioning scores were mostly in the fair-to-poor range. Similarly, satisfaction with care was not related to functional status and anxiety among patients who were admitted to a rehabilitation unit after hip or knee surgery [3]. A study on older hospitalized medical patients found that patient health status at discharge, rather than degree of improvement or decline, was related to treatment satisfaction [4]. Thus, patients with similar health status at discharge reported similar levels of satisfaction, regardless of whether their status had improved, declined, or stabilized since their admission.

A survey of adolescents who underwent surgery for idiopathic scoliosis found that 73 percent were satisfied with the cosmetic results. Preoperative psychological difficulties and unrealistic expectations regarding the cosmetic results were associated with patient dissatisfaction or neutrality [5]. Research on patients with chronic pain indicated that expectation of pain relief following total hip and knee arthroplasty was related to subsequent functional ability [6]. This finding suggests that patient expectation may influence treatment outcome; however, patient satisfaction with and effectiveness of pain services were not assessed in this study. In another study of patients who underwent automated percutaneous lumbar discectomy, satisfaction was not related to pain relief but age was; specifically, younger patients reported better outcomes [7].

Multiple studies of postoperative patients have examined the relation of reported pain intensity to satisfaction with pain treatment. Small but significant negative correlations between pain intensity and treatment satisfaction have been found in surveys of postoperative and other hospitalized patients [8-11]. Typically, patients report satisfaction with treatment even when pain management is not optimal [11-18]. Perceived control over pain, communication with providers, and effectiveness of pain medications are other variables that have been associated with treatment satisfaction $[8,11]$.

Several studies of patients with chronic pain have examined the relation of pain intensity and satisfaction to pain management. An evaluation of patient perceptions in a multidisciplinary pain program found that patient ratings of the helpfulness of the entire program correlated significantly with patient ratings of decreased pain intensity, reduced disability, and improved coping [19-20]. A global rating of treatment satisfaction was significantly correlated with changes in pain, depression, and anxiety in a small sample of patients who attended a specialty clinic for pain management [21]. The strongest predictors of satisfaction, however, were patient perceptions of improvement in daily activity, the belief that their evaluation was complete, and the belief that clinic procedures were well explained. A study found that more than 75 percent of cancer patients with pain were satisfied or very satisfied with their overall pain management [22]. The significant predictors of satisfaction included the patient's report of long-term pain relief, whether or not the patient was told that pain treatment was an important goal, and the patient's willingness to take opioids.

In summary, some studies have found statistically significant but modest associations between patient satisfaction or perceived effectiveness of care and measures of treatment outcome among patients with pain. These mixed findings may be due, in part, to differences in the patient populations and variability in the tools used for assessing satisfaction. Moreover, to date, researchers have tended to examine associations between a very limited number of satisfaction ratings and treatment outcome variables in any one study. Given the frequent use and simplicity of satisfaction ratings, further exploration of their potential validity for assessing treatment outcome makes sense.

The present study explored the association between patient satisfaction ratings and treatment outcome with the use of multiple measures. Based on our literature review, we hypothesized that patient ratings of treatment satisfaction and effectiveness would be significantly associated with pre- to posttreatment changes in pain intensity, pain interference, and disability. We also hypothesized that posttreatment ratings of change in pain condition and quality of life (QOL) would be significantly related to pre- to posttreatment changes in pain intensity and pain interference.

\section{METHODS}

\section{Participants}

This study involved patients with chronic noncancer pain who were referred to the Integrated Pain Management Program (IPMP) of the Michael E. DeBakey Department of Veterans Affairs Medical Center (VAMC), a tertiary teaching hospital. The IPMP is a multidisciplinary outpatient pain assessment, consulting, and treatment 
program that receives referrals from various surgical, medical, and psychiatric departments within the hospital. Patients referred to the IPMP have often received pain treatments in other VAMC clinics. Therefore, they had typically been experiencing pain for months, if not years, prior to their IPMP referrals. Prior to their initial assessment in the program, patients completed a packet of selfreport questionnaires. Instructions for completing the questionnaires were identical to those given by the authors who developed the measures. The questionnaire packet was mailed to patients along with a cover letter that explained the goals of the program. Patients were informed that the questionnaires were primarily for clinical purposes but the data might also be used for program evaluation and research. Prior to analyzing the data, we obtained approval from the Baylor University Institutional Review Board.

\section{Treatment Program}

The IPMP is a joint effort of the anesthesiology, physical medicine and rehabilitation (PM\&R), and psychology departments. A pain anesthesiologist, a pain psychologist, and a physiatrist interviewed all patients. An individualized treatment plan was developed for each patient at a multidisciplinary conference. A typical plan included various combinations of pain medication; nerve blocks; PM\&R services, such as physical therapy and transcutaneous electrical nerve stimulation; biofeedback; patient education/support groups; and psychological counseling. All referred patients agreed to participate in the program. From 6 to 9 months after the implementation of the treatment plan, the patients were seen for a review of their treatment progress. Approximately 50 percent of the treated patients attended a review session. As a part of this follow-up interview, they were asked to complete a ratingof-services form and a questionnaire packet identical to the initial assessment packet so that their responses to the outcome assessment tools could be compared across time.

\section{Sample}

A total of 122 patients who attended a review session and completed the treatment and assessment measures were included in the data analysis. The average patient age was 50.8 (standard deviation $=11.4$, range $=22-82$ ) . Most (85.3\%) were at least high school graduates or had received a general equivalency diploma (GED), 12.8 percent were college graduates, and 1.9 percent had less than a high school education. The majority of patients were male (90.3\%). Although most were white (62.4\%), 22.6 percent were African American, 4.6 percent were Hispanic, 0.5 percent were other, and 9.9 percent did not respond to this item. Approximately half (52.8\%) of the patients were married. The primary pain sites were the back (39\%), leg or arm (32\%), neck and shoulder (19\%), and other (10\%). Almost half (48\%) of the patients were already receiving disability compensation for a painrelated condition, and more than half (58\%) indicated that they had been disabled by pain for more than 5 years.

\section{Measures}

The measures of pain and functioning analyzed were the Center for Epidemiologic Studies Depression Scale (CES-D) for assessment of depression, the RolandMorris Disability Questionnaire (RMDQ) for assessment of disability, the Interference scale of the West HavenYale Multidimensional Pain Inventory (WHYMPI) for assessment of how pain has interfered with the patient's life, and the Pain Severity scale of the WHYMPI.

The CES-D was developed for assessment of the presence and severity of depressive symptomatology in the general population [23]. The CES-D includes 20 items that are answered on a 4-point scale $(0=$ rarely, $3=$ most of the time) and results in scores that range from 0 to 60 (higher scores indicate greater depressive symptomatology). The CES-D has been widely used in pain research [24-26] and has adequate reliability and convergent validity [23].

The RMDQ [27], derived from the Sickness Impact Profile, was developed for assessment of disability associated with back pain. RMDQ items focus almost exclusively on the physical dimensions of disability [28]. Subjects indicate which, if any, of 24 statements describe them today and are related to their pain. (For example, "I stay at home most of the time because of my pain.") Scores range from 0 to 24, with higher scores indicating greater disability. Research supports the validity and reliability of the scale for assessing disability among persons with mixed chronic pain problems [29] and lowback pain $[28,30]$.

The Interference scale of the WHYMPI consists of 11 items that assess how pain has interfered with daily activities and functioning, including the ability to work, enjoy family, participate in social and recreational activities, and perform household chores [31]. Scale scores range from 0 to 6, with higher scores i3.3icating greater perceived interference with daily functioning. The Interference scale 
is reported to have an excellent internal consistency of 0.90 and a test-retest stability of 0.86 [31].

The Pain Severity scale of the WHYMPI was used for assessment of pain severity [31]. This scale consists of three items that assess pain intensity and suffering. Scores range from 0 to 6 , with higher scores indicating greater pain intensity and suffering. The scale is reported to have an internal consistency of 0.72 and a test-retest stability of 0.82 [31].

We obtained ratings of services by asking the patients to rate on an ordinal scale such factors as satisfaction with services, effectiveness of services received, and changes in pain condition and QOL as a result of treatment received. Thus, we targeted not only patients' treatment satisfaction but also their perceptions of treatment efficacy and of changes in pain condition and QOL following treatment. Patients were asked to rate their overall satisfaction with pain services as "low," "medium," or "high"; the overall effectiveness of received pain services as "not effective," "somewhat effective," or "very effective;" changes in pain condition as "worsened," "unchanged," or "improved;" and changes in QOL as "worsened," "unchanged," or "improved."

\section{Data Analyses}

We performed data analyses to address the following questions:

1. Did the patients improve on any or all of the four measures of pain and functioning after receiving the treatment services?

2. Were posttreatment service ratings and pre- to posttreatment changes in pain and functioning significantly associated?

To answer question 1 , we performed a multivariate Hotelling analysis $\left(T^{2}\right)$ comparing the pre- and posttreatment values of the four psychometric variables. We then used a series of Student $t$-tests to compare the pre- and posttreatment values for each variable. To evaluate question 2, we used multivariate analyses of variance (MANOVAs) to compare changes in the four psychometric variables across the three levels of each service rating. If the MANOVA was significant, we used analyses of variance (ANOVAs) to address question 2 . When $F$-values were significant, pairwise contrasts were performed with the Tukey criteria. Missing data were replaced by the group mean. The percentage of missing values was 6.6 percent for the service ratings and 2.4 percent for the measures of pain and function. We ran two sets of analyses: one set with data from participants who provided complete data (i.e., nonreplaced data) and one set with missing data replaced by the group mean. Because the results of the two analytic approaches did not differ, we report the analyses with missing data replaced by the group mean to facilitate comparisons among the results.

\section{RESULTS}

Table 1 presents the patient ratings of satisfaction with treatment, effectiveness of treatment, change in pain condition, and change in QOL after treatment. Approximately 62 percent of the patients expressed medium or high satisfaction with the services received, and approximately 69 percent rated the pain services as somewhat or very effective. About 67 percent rated their pain as improved or unchanged following treatment, and 68 percent rated their QOL as improved or unchanged.

Table 2 shows the mean scores on the outcome measures at pre- and posttreatment. Prior to the individual $t$-tests, we used a multivariate Hotelling $\left(T^{2}\right)$ analysis to compare the pre- and posttreatment values of the four psychometric variables. Significance was $p<0.001$ based on the Wilks $\Lambda$ statistic. The individual $t$-tests revealed that patients reported significant pre- to posttreatment decreases in pain severity, pain interference, and depression. Effect sizes of change scores as calculated from results of the individual $t$-tests were $-0.40,-0.26,-0.32$,

Table 1.

Patient ratings ( $n=122)$ of services provided in multidisciplinary pain treatment program.

\begin{tabular}{llc}
\hline \multicolumn{1}{c}{ Item } & \multicolumn{1}{c}{ Scale } & Response (\%) \\
\hline Overall Satisfaction & Low & 37.7 \\
with Pain Services & Medium & 43.4 \\
& High & 18.9 \\
Overall Effectiveness & Not effective & 31.1 \\
of Pain Services & Somewhat effective & 48.4 \\
& Very effective & 20.5 \\
Change in Pain & Worsened & 32.8 \\
Condition & Unchanged & 33.6 \\
& Improved & 33.6 \\
Change in Quality of & Worsened & 32.0 \\
Life & Unchanged & 37.7 \\
& Improved & 30.3 \\
\hline \hline
\end{tabular}


and -0.11 for pain severity, pain interference, depression, and disability, respectively. These effect sizes indicate that, based on generally accepted criteria [32], painseverity change had a medium effect size, while interference and depression changes had small effect sizes.

MANOVAs were used for comparing changes in the four psychometric outcome variables (pain severity, pain interference, depression, and disability) across the three levels of each service rating. The multivariate $p$-values using the Wilks $\Lambda$ test were (1) satisfaction with pain services, $F_{4,117}=4.44, p=0.002$; (2) effectiveness of pain services, $F_{4,117}=4.04, p=0.004$; (3) change in pain condition, $F_{4,117}=7.30, p>0.0001$; and (4) change in quality of life, $F_{4,117}=7.73, p<0.0001$.

We used these results to justify separate ANOVAs. Tables 3-6 show the results of one-way ANOVAs of the pre- to posttreatment change scores of the four outcome variables across the three service-rating levels for satisfaction (Table 3), effectiveness (Table 4), pain condition (Table 5), and quality of life (Table 6). All analyses used values from the same 122 patients in Table 2 . Changes in pain severity and pain interference consistently had the

Table 2.

Comparison of participant $(n=122)$ pre- and posttreatment mean values on four pain and functioning measures.

\begin{tabular}{|c|c|c|c|c|c|c|c|}
\hline Measure & $\begin{array}{c}\text { Pretreatment } \\
(\text { Mean } \pm \text { SD) }\end{array}$ & $\begin{array}{c}\text { Posttreatment } \\
\text { (Mean } \pm \text { SD) }\end{array}$ & Difference & SE & $t$-test ${ }^{*}$ & $\begin{array}{c}\text { Effect } \\
\text { Size }\end{array}$ & p-Value \\
\hline Pain Severity (WHYMPI) & $4.99 \pm 0.94$ & $4.56 \pm 1.22$ & -0.42 & 0.10 & -4.46 & -0.40 & $<0.002$ \\
\hline Depression (CESD) & $28.26 \pm 11.86$ & $25.52 \pm 11.99$ & -2.74 & 0.78 & -3.52 & -0.32 & $<0.001$ \\
\hline Disability (RMDQ) & $16.24 \pm 4.98$ & $15.82 \pm 5.76$ & -0.42 & 0.34 & -1.21 & -0.11 & 0.230 \\
\hline
\end{tabular}

*Degrees of freedom $=2,119$.

CESD = Center for Epidemiologic Studies Depression Scale, RMDQ = Roland-Morris Disability Questionnaire, $\mathrm{SD}=$ standard deviation, $\mathrm{SE}=$ standard error, WHYMPI = West Haven-Yale Multidimensional Pain Inventory.

Table 3.

Analysis of variance of participant self-reported satisfaction with pain services (low, $n=46$; medium, $n=53$; high, $n=23$ ) with changes in outcome variables.

\begin{tabular}{|c|c|c|c|c|c|c|}
\hline Change in Outcome Variable ${ }^{*}$ & $\begin{array}{c}\text { Low } \\
(\text { Mean } \pm \text { SD) }\end{array}$ & $\begin{array}{c}\text { Medium } \\
(\text { Mean } \pm \text { SD) }\end{array}$ & $\begin{array}{c}\text { High } \\
(\text { Mean } \pm \text { SD) }\end{array}$ & $\boldsymbol{F}^{\dagger}$ & $p$-Value & Effect Size \\
\hline Pain Severity & $-0.09_{a} \pm 0.69$ & $-0.41_{\mathrm{a}} \pm 0.99$ & $-1.13_{b} \pm 1.43$ & 8.48 & $<0.001$ & 0.46 \\
\hline Pain Interference & $-0.01_{\mathrm{a}} \pm 0.69$ & $-0.23_{\mathrm{a}, \mathrm{b}} \pm 0.85$ & $-0.81_{b} \pm 1.53$ & 5.35 & 0.006 & 0.35 \\
\hline Depression & $-2.73_{\mathrm{a}} \pm 8.01$ & $-2.73_{a} \pm 8.48$ & $-2.79_{a} \pm 10.27$ & 0.00 & 1.00 & 0.00 \\
\hline Disability & $0.13_{a} \pm 3.69$ & $-0.57 \mathrm{a} \pm 3.67$ & $-1.17_{\mathrm{a}} \pm 4.29$ & 0.97 & 0.380 & 0.00 \\
\hline
\end{tabular}

${ }^{*}$ For each outcome variable, mean values with differing subscripts are statistically significantly different, $p<0.05$.

${ }^{\dagger}$ Degrees of freedom $=2,119$.

$F$ = variance ratio score, $\mathrm{SD}=$ standard deviation.

Table 4.

Analysis of variance of participant self-reported effectiveness of pain services (not effective, $n=38$; somewhat effective, $n=59$; very effective, $n=25)$ with changes in outcome variables.

\begin{tabular}{|c|c|c|c|c|c|c|}
\hline Change in Outcome Variable* & $\begin{array}{l}\text { Not Effective } \\
\text { (Mean } \pm \text { SD) }\end{array}$ & $\begin{array}{c}\text { Somewhat Effective } \\
\text { (Mean } \pm \text { SD) }\end{array}$ & $\begin{array}{c}\text { Very Effective } \\
\text { (Mean } \pm \text { SD) }\end{array}$ & $\boldsymbol{F}^{\dagger}$ & $p$-Value & Effect Size \\
\hline Pain Severity & $-0.18_{a} \pm 0.89$ & $-0.28_{a} \pm 0.80$ & $-1.14_{b} \pm 1.46$ & 8.20 & $<0.001$ & 0.45 \\
\hline Pain Interference & $0.11_{\mathrm{a}} \pm 0.82$ & $-0.28_{\mathrm{a}, \mathrm{b}} \pm 0.80$ & $-0.76_{b} \pm 1.41$ & 6.29 & 0.003 & 0.38 \\
\hline Depression & $-1.62 \mathrm{a} \pm 9.51$ & $-3.25 a \pm 6.92$ & $-3.25 a \pm 10.71$ & 0.63 & 0.630 & 0.00 \\
\hline Disability & $0.29_{a} \pm 3.93$ & $-0.17 \mathrm{a}, \mathrm{b} \pm 3.05$ & $-2.08_{b} \pm 4.76$ & 3.30 & 0.040 & 0.25 \\
\hline
\end{tabular}

*For each outcome variable, mean values with differing subscripts are statistically significantly different, $p<0.05$.

${ }^{\dagger}$ Degrees of freedom $=2,119$.

$F=$ variance ratio score, $\mathrm{SD}=$ standard deviation . 
Table 5.

Analysis of variance of participant self-rated change in pain condition (worsened, $n=40$; unchanged, $n=41$; improved, $n=41$ ) with changes in outcome variables.

\begin{tabular}{|c|c|c|c|c|c|c|}
\hline Change in Outcome Variable* & $\begin{array}{c}\text { Worsened } \\
(\text { Mean } \pm \text { SD) }\end{array}$ & $\begin{array}{l}\text { Unchanged } \\
(\text { Mean } \pm \text { SD) }\end{array}$ & $\begin{array}{c}\text { Improved } \\
(\text { Mean } \pm \text { SD) }\end{array}$ & $\boldsymbol{F}^{\dagger}$ & $p$-Value & Effect Size \\
\hline Pain Severity & $-0.03_{a} \pm 0.74$ & $-0.30_{a} \pm 0.85$ & $-1.00_{b} \pm 1.23$ & 12.10 & $<0.001$ & 0.52 \\
\hline Pain Interference & $0.09_{a} \pm 0.70$ & $-0.86_{a} \pm 0.75$ & $-0.77_{b} \pm 1.25$ & 9.65 & $<0.001$ & 0.46 \\
\hline Depression & $-0.21_{a} \pm 8.13$ & $-3.26_{a, b} \pm 7.64$ & $-4.69_{b} \pm 9.50$ & 2.95 & 0.056 & 0.22 \\
\hline Disability & $0.38_{a} \pm 2.82$ & $0.49_{a} \pm 3.80$ & $-2.10_{b} \pm 4.14$ & 6.60 & 0.002 & 0.37 \\
\hline
\end{tabular}

${ }^{*}$ For each outcome variable, mean values with differing subscripts are statistically significantly different, $p<0.05$.

${ }^{\dagger}$ Degrees of freedom $=2,119$.

$F=$ variance ratio score, $\mathrm{SD}=$ standard deviation .

Table 6.

Analysis of variance of participant self-rated change in quality of life (worsened, $n=39$; unchanged, $n=46$; improved, $n=37$ ) with change in outcome variables.

\begin{tabular}{ccccrrr}
\hline Change in Outcome Variable $^{*}$ & $\begin{array}{c}\text { Worsened } \\
\text { (Mean } \pm \text { SD) }\end{array}$ & $\begin{array}{c}\text { Unchanged } \\
\text { (Mean } \pm \text { SD) }\end{array}$ & $\begin{array}{c}\text { Improved } \\
\text { (Mean } \pm \text { SD) }\end{array}$ & $\boldsymbol{F}^{\dagger}$ & $\boldsymbol{p}^{\text {-Value }}$ & Effect Size \\
\hline Pain Severity & $0.15_{\mathrm{a}} \pm 0.79$ & $-0.46_{\mathrm{b}} \pm 0.68$ & $-0.98_{\mathrm{c}} \pm 1.34$ & 13.51 & $<0.001$ & 0.56 \\
Pain Interference & $0.12_{\mathrm{a}} \pm 0.73$ & $-0.24_{\mathrm{a}, \mathrm{b}} \pm 0.84$ & $-0.67_{\mathrm{b}} \pm 1.26$ & 6.40 & 0.002 & 0.37 \\
Depression & $-0.56_{\mathrm{a}} \pm 8.42$ & $-2.72_{\mathrm{a}} \pm 7.48$ & $-5.06_{\mathrm{a}} \pm 9.65$ & 2.68 & 0.073 & 0.20 \\
Disability & $1.00_{\mathrm{a}} \pm 3.00$ & $-0.70_{\mathrm{a}, \mathrm{b}} \pm 3.75$ & $-1.57_{\mathrm{b}} \pm 4.23$ & 4.82 & 0.010 & 0.31 \\
\hline
\end{tabular}

${ }^{*}$ For each outcome variable, mean values with differing subscripts are statistically significantly different, $p<0.05$.

${ }^{\dagger}$ Degrees of freedom $=2,119$.

$F=$ variance ratio score, $\mathrm{SD}=$ standard deviation.

greatest association with the service ratings of treatment satisfaction, effectiveness, change in pain condition, and quality of life, with highly significant $p$-values in all four variables. The patients' self-reported changes in disability had significant associations with service ratings of treatment effectiveness, change in pain condition, and change in quality of life. Change in depression was not significantly related to any of the service ratings. Effect sizes for pain severity were medium to large [32] for all the service ratings; effect sizes for pain interference and disability ranged from small to medium.

Tables 3-6 also present the pairwise contrasts that were performed among the outcome-variable change scores for the three levels of service ratings. In all cases of significant $F$-values, the contrast between the low and high levels of the service rating was significant. Patients with high ratings of satisfaction with pain services reported greater pre- to posttreatment decreases in pain severity and pain interference than patients with low ratings. Patients with high ratings of pain services and QOL reported significantly more improvements in pain severity, interference, and disability than patients with low ratings. The patients who reported their pain condition as improved also demonstrated more improvements in pain severity, interference, depression, and disability than patients who reported their pain condition as worsened. Additional significant comparisons included the contrasts between the middle and high values of all service ratings for pain severity, and the middle and high service ratings of pain condition for pain interference. Patients with higher service ratings reported greater decreases in pain severity and pain interference than patients with lower ratings. The contrast between low and middle levels of QOL was significant for pain severity, with patients with higher ratings of improvement in quality of life reporting greater decreases in pain severity.

We studied whether race/ethnicity (white or nonwhite), marital status (married or not), and education (at least high school/GED or less than high school) were related to the posttreatment psychometric and service ratings. Previous research has indicated that these demographic variables may influence the outcome of multidisciplinary painmanagement programs $[3,28,33]$. Among the statistically significant relationships was one between disability and race/ethnicity; white patients $(n=62)$ had lower mean ratings than nonwhite patients $(n=31)$, with mean values of 14.97 versus 18.45, respectively, $\left(t_{91}=-3.23, p=0.002\right)$. 
All four psychometric outcome variables had lower mean values for patients with at least a high school education/GED $(n=93)$ compared with patients with less than a high school education $(n=16)$; specifically, mean pain severity was 4.48 versus 5.54 , respectively $\left(t_{107}=-3.29\right.$, $p=0.001$ ); mean pain interference was 4.65 versus 5.40, respectively $\left(t_{107}=-2.61, p=0.01\right)$; mean disability was 15.59 versus 19.19 , respectively $\left(t_{107}=-2.58, p=0.01\right)$; and mean depression was 10.93 versus 13.31 , respectively $\left(t_{107}=-3.77, p=0.0003\right)$.

Changes in QOL and education were also significantly related. Of the 93 patients with at least a high school education/GED, 28 percent rated their change in QOL as worsened, 39 percent as unchanged, and 33 percent as improved. In comparison, among the 16 patients with less than a high school education, 62 percent rated their change in QOL as worsened, 18 percent as unchanged, and 18 percent as improved $\left(\chi^{2}{ }_{1}=5.09, p=\right.$ 0.02).

\section{DISCUSSION}

The results indicate significant improvements on ratings of pain severity, pain interference, and depression from pre- to posttreatment among the patients in this multidisciplinary pain-management program. However, no significant change in disability (functional status) was found. Analysis of effect sizes indicated a medium effect for pain severity and small effects for pain interference and depression.

Patient ratings of satisfaction with and effectiveness of treatment were less favorable than those found in previous studies of multidisciplinary pain programs [19-21]; 38 percent of the patients gave low satisfaction ratings and 31 percent gave low effectiveness ratings. Similarly, most patients did not rate changes in pain condition and QOL favorably; only 34 percent reported improved pain condition and 30 percent reported improved QOL. These results may be partly due to the severity, chronicity, and heterogeneity of the pain conditions represented in the study sample. Furthermore, the patients did not receive a standard set of interventions; rather, the interventions were individualized and implemented by different staff in different departments. For example, some patients received epidural injections, while others received biofeedback, physical therapy, or a combination of various treatments. This individualized approach to pain manage- ment has not been compared with a more standardized multidisciplinary program and its efficacy may be limited.

In the present study, the patient ratings of services (satisfaction, effectiveness, changes in pain condition, and QOL) were significantly associated with changes in several outcome measures. As predicted, pre- to posttreatment changes in pain severity and pain interference were significantly associated with service ratings of satisfaction, effectiveness, change in pain condition, and QOL. Change in the patients' self-reported disability from pre- to posttreatment was significantly related to ratings of treatment effectiveness and changes in pain condition and QOL. These findings support the need for pain-management programs that focus on decreasing pain intensity, pain interference, and disability. Although mean depression levels were significantly reduced at posttreatment, the patients' pre- to posttreatment change in self-reported depression was not significantly associated with any of the service ratings. This finding suggests that patients' evaluations of a pain-treatment program are related to perceptions of improvements in pain and daily function rather than mood. Also, given that mood change was not a focus of treatment, individuals whose mood did not improve but whose pain decreased did not feel that treatment was any less successful than individuals whose mood did improve.

The patients' race/ethnicity was significantly associated with self-report of disability. The nonwhite patients, primarily African American, reported greater disability than the white patients. Previous research has documented that minority patients are at risk for undertreatment of acute, postoperative, and cancer pain [34]. Very little research has examined the possible influence of race/ ethnicity on treatment outcomes for patients with chronic noncancer pain. The results have been mixed; some descriptive studies indicated no significant differences in treatment outcomes as a function of race/ethnicity [35] and others found that minority patients were at risk for less positive outcomes, including greater disability [3334]. In the present study, the white and nonwhite patients did not differ in their ratings of satisfaction, effectiveness, or changes in pain condition and QOL. Longitudinal research is needed for investigating the adequacy of pain treatment programs for African-American and other racial/ethnic minority patients.

Level of education was significantly associated with posttreatment pain severity, pain interference, depression, and disability. Patients with at least a high school 
education/GED reported more positive outcomes than individuals with less than a high school education. In addition, patients with less than a high school education were less likely to report a positive change in QOL. The association between education and treatment outcomes may reflect the relationship of education to socioeconomic status and daily function. In a national survey of individuals with back pain, Deyo and Centor found that education strongly predicted functional status [28]. Additional research must evaluate the impact of education on treatment outcomes and patient ratings of services for chronic pain.

Several limitations of this study should be noted. First, the patients in this study were primarily male veterans with relatively severe and chronic pain conditions. Moreover, only a portion of those who received treatment came to the follow-up session and completed the posttreatment questionnaire packet. Thus, dissatisfied patients might have been overrepresented in the dropout group. For these reasons, the findings should be generalized cautiously. Second, response bias may have influenced the responses of patients who completed the outcome assessment. A member of the multidisciplinary treatment team typically administered the outcome measures. Third, because the current results are correlational, they do not represent causal relationships between the patient ratings of services and treatment outcome as reflected by the psychometric measures used. The pre- to posttreatment changes in the outcome measures may be related to multiple factors that include random effects, regression to the mean, and the pain interventions. A fourth limitation is the reliance on self-report measures for assessment of functioning, especially with regard to disability. An additional measure of disability, such as a clinician's rating of functional status would have been desirable. A related limitation is the lack of information on the patients' pain diagnoses and specific treatment plans. These variables possibly influence patient ratings of services received in a multidisciplinary pain-management program.

Another limitation of our study is the limited information about the psychometric properties of the treatment-satisfaction measures that were used. The present results, however, provide evidence of the construct validity of the measures. The patient ratings of treatment satisfaction, effectiveness, and changes in pain condition and QOL were highly associated with changes in pain intensity and pain interference following treatment. Furthermore, the ratings of effectiveness and changes in pain condition and QOL were significantly associated with posttreatment disability. Thus, patients with lower levels of pain, pain interference, and disability at posttreatment were more likely to rate the pain-management services positively than patients with less favorable outcomes. We need additional research to further explore the reliability and validity of our treatment-satisfaction measures and compare them with alternative measures. The construct of satisfaction with pain-management services has not been well defined. Our measure included four possible components but did not assess other possible domains, such as satisfaction with service providers, evaluations of analgesic medications, or satisfaction with information and explanations provided in the treatment program.

\section{CONCLUSIONS}

Most pain-management programs are mandated to assess patient satisfaction with services. Despite the limitations of our study, the findings suggest that, unlike general surveys of patient satisfaction with services that focus on staff courtesy, length of waiting time, and other treatment-related variables, patient ratings of satisfaction and effectiveness of the program could be used as indices of treatment outcome. For instance, in this study, patients were first asked to rate satisfaction with and effectiveness of treatment and then to rate changes in pain condition and quality of life. Hence, the rating questions were asked in the context of "Did the interventions work?" as opposed to "Were you treated well?" Additional research is needed for further evaluating this approach to service ratings and for determining whether this approach produces a stronger link between patient ratings of satisfaction and treatment outcome than global ratings alone. Our findings also indicate that different types of satisfaction ratings may reflect changes in different factors that are commonly targeted in pain-management programs. The results support the importance of assessing not just satisfaction with treatment but also perceived changes in functioning, pain, and overall QOL after pain-management interventions.

\section{ACKNOWLEDGMENTS}

This material was unfunded at the time of manuscript preparation.

The authors have declared that no competing interests exist. 


\section{REFERENCES}

1. Branch WT Jr, Kern D, Haidet P, Weissmann P, Gracey CF, Mitchell G, Inui T. The patient-physician relationship. Teaching the human dimensions of care in clinical settings. JAMA. 2001;286(9):1067-74. [PMID: 11559292]

2. Tomaino MM, Miller RJ, Burton RI. Outcome assessment following limited wrist fusion: objective wrist scoring versus patient satisfaction. Contemp Orthop. 1994;28(5):403-10. [PMID: 10147142]

3. Franchignoni F, Ottonello M, Benevolo E, Tesio L. Satisfaction with hospital rehabilitation: is it related to life satisfaction, functional status, age or education? J Rehabil Med. 2002;34(3):105-8. [PMID: 12395936]

4. Covinsky KE, Rosenthal GE, Chren MM, Justice AC, Fortinsky RH, Palmer RM, Landefeld CS. The relation between health status changes and patient satisfaction in older hospitalized medical patients. J Gen Intern Med. 1998;13(4): 223-29. [PMID: 9565384]

5. Koch KD, Buchanan R, Birch JG, Morton AA, Gatchel RJ, Browne RH. Adolescents undergoing surgery for idiopathic scoliosis: how physical and psychological characteristics relate to patient satisfaction with the cosmetic result. Spine. 2001;26(19):2119-24. [PMID: 11698890]

6. Mahomed NN, Liang MH, Cook EF, Daltroy LH, Fortin PR, Fossel AH, Katz JN. The importance of patient expectations in predicting functional outcomes after total joint arthroplasty. J Rheumatol. 2002;29(6):1273-79. [PMID: 12064846]

7. Bernd L, Schiltenwolf M, Mau H, Schindele S. No indications for percutaneous lumbar discectomy? Int Orthop. 1997;21(3):164-68. [PMID: 9266295]

8. Carlson J, Youngblood R, Dalton JA, Blau W, Lindley C. Is patient satisfaction a legitimate outcome of pain management? J Pain Symptom Manage. 2003;25(3):264-75. [PMID: 12614961]

9. McNeill JA, Sherwood GD, Starck PL, Thompson CJ. Assessing clinical outcomes: patient satisfaction with pain management. J Pain Symptom Manage. 1998;16(1):29-40. [PMID: 9707655]

10. Pellino TA, Ward SE. Perceived control mediates the relationship between pain severity and patient satisfaction. J Pain Symptom Manage. 1998;15(2):110-16. [PMID: 9494309]

11. Ward SE, Gordon DB. Patient satisfaction and pain severity as outcomes in pain management: a longitudinal view of one setting's experience. J Pain Symptom Manage. 1996; 11(4):242-51. [PMID: 8869459]

12. Beauregard L, Pomp A, Choiniere M. Severity and impact of pain after day-surgery. Can J Anaesth. 1998;45(4):304-11. [PMID: 9597202]

13. Carroll KC, Atkins PJ, Herold GR, Mlcek CA, Shively M, Clopton P, Glaser DN. Pain assessment and management in critically ill postoperative and trauma patients: a multisite study. Am J Crit Care. 1999;8(2):105-17. [PMID: 10071701]

14. Comley AL, DeMeyer E. Assessing patient satisfaction with pain management through a continuous quality improvement effort. J Pain Symptom Manage. 2001;21(1): 27-40. [PMID: 11223312]

15. Jamison RN, Ross MJ, Hoopman P, Griffin F, Levy J, Daly $\mathrm{M}$, Schaffer JL. Assessment of postoperative pain management: patient satisfaction and perceived helpfulness. Clin J Pain. 1997;13(3):229-36. [PMID: 9303255]

16. Miaskowski C, Nichols R, Brody R, Synold T. Assessment of patient satisfaction utilizing the American Pain Society's Quality Assurance Standards on acute and cancer-related pain. J Pain Symptom Manage. 1994;9(1):5-11. [PMID: 8169462]

17. Myles PS, Williams DL, Hendrata M, Anderson H, Weeks AM. Patient satisfaction after anaesthesia and surgery: results of a prospective survey of 10,811 patients. $\mathrm{Br} \mathrm{J}$ Anaesth. 2000;84(1):6-10. [PMID: 10740539]

18. Starck PL, Adams J, Sherwood GD, Thompson CJ. Development of a pain management report card for an acute care setting. Adv Pract Nurs Q. 1997;3(2):57-63. [PMID: 9432454]

19. Chapman SL, Jamison RN, Sanders SH. Treatment Helpfulness Questionnaire: a measure of patient satisfaction with treatment modalities provided in chronic pain management programs. Pain. 1996;68(2-3):349-61.

[PMID: 9121824]

20. Chapman SL, Jamison RN, Sanders SH, Lyman DR, Lynch NT. Perceived treatment helpfulness and cost in chronic pain rehabilitation. Clin J Pain. 2000;16(2):169-77. [PMID: 10870730]

21. McCracken LM, Evon D, Karapas ET. Satisfaction with treatment for chronic pain in a specialty service: preliminary prospective results. Eur J Pain. 2002;6(5):387-93. [PMID: 12160513]

22. Dawson R, Spross JA, Jablonski ES, Hoyer DR, Sellers DE, Solomon MZ. Probing the paradox of patients' satisfaction with inadequate pain management. J Pain Symptom Manage. 2002;23(3):211-20. [PMID: 11888719]

23. Radloff LS, Locke B. The community mental health assessment survey and the CES-D scale. In: Weissman M, Myers J, Ross C, editors. Community surveys. Rutgers (NJ): Rutgers University Press; 1977.

24. Brown GK. A causal analysis of chronic pain and depression. J Abnorm Psychol. 1990;99(2):127-37. [PMID: 2348006]

25. Jensen MP, Karoly P. Control beliefs, coping efforts, and adjustment to chronic pain. J Consult Clin Psychol. 1991; 59(3):431-38. [PMID: 2071728]

26. Tan G, Jensen MP, Robinson-Whelen S, Thornby JI, Monga TN. Coping with chronic pain: a comparison of two measures. Pain. 2001;90(1-2):127-33. [PMID: 11166978] 
27. Roland M, Morris R. A study of the natural history of back pain. Part I: Development of a reliable and sensitive measure of disability in low-back pain. Spine. 1983;8(2):141-44. [PMID: 6222486]

28. Deyo RA, Centor RM. Assessing the responsiveness of functional scales to clinical change: an analogy to diagnostic test performance. J Chronic Dis. 1986;39(11):897-906. [PMID: 2947907]

29. Jensen MP, Strom SE, Turner JA, Romano JM. Validity of the Sickness Impact Profile Roland scale as a measure of dysfunction in chronic pain patients. Pain. 1992;50(2):157-62. [PMID: 1408311]

30. Lanier DC, Stockton P. Clinical predictors of outcome of acute episodes of low back pain. J Fam Pract. 1988;27(5): 483-89. [PMID: 2973513]

31. Kerns RD, Turk DC, Rudy TE. The West Haven-Yale Multidimensional Pain Inventory (WHYMPI). Pain. 1985;23(4): 345-56. [PMID: 4088697]
32. Cohen J. Statistical power analysis for the behavioral sciences. 2nd ed. Hillsdale (NJ): Erlbaum; 1988.

33. Green CR, Ndao-Brumblay SK, Nagrant AM, Baker TA, Rothman E. Race, age, and gender influences among clusters of African American and white patients with chronic pain. J Pain. 2004;5(3):171-82. [PMID: 15106130]

34. Green CR, Anderson KO, Baker TA, Campbell LC, Decker S, Fillingim RB, Kaloukalani DA, Lasch KE, Myers C, Tait RC, Todd KH, Vallerand AH. The unequal burden of pain: confronting racial and ethnic disparities in pain. Pain Med. 2003;4(3):277-94. [PMID: 12974827]

35. Novy DM, Nelson DV, Hetzel RD, Squitieri P, Kennington M. Coping with chronic pain: sources of intrinsic and contextual variability. J Behav Med. 1998;21(1):19-34. [PMID: 9547420]

Submitted for publication October 5, 2004. Accepted in revised form December 30, 2005. 